\title{
Full Configuration Interaction Computer Simulation Study of the Thermodynamic and Kinetic Stability of Hydrated Dielectrons
}

\author{
Ross E. Larsen and Benjamin J. Schwartz* \\ Department of Chemistry and Biochemistry, University of California, Los Angeles, California 90095-1569
}

Received: August 17, 2005; In Final Form: October 28, 2005

\begin{abstract}
The hydrated electron is a unique solvent-supported state comprised of an excess electron that is confined to a cavity by the surrounding water. Theoretical studies have suggested that two-electron solvent-supported states also can be formed; in particular, simulations indicate that two excess electrons could pair up and occupy a single cavity, forming a so-called hydrated dielectron. Although hydrated dielectrons have not been observed directly by experiment, their existence has been posited to explain the lack of an ionic strength effect in hydrated electron bimolecular annihilation [Schmidt, K. H.; Bartels, D. M. Chem. Phys. 1995, 190, 145]. To determine whether dielectrons may be created in the laboratory, we use thermodynamic integration (TI), combined with mixed quantum/classical molecular dynamics simulation, to examine the thermodynamic stability of hydrated electrons and dielectrons. For the dielectron calculations, we solve the two-electron quantum problem using full configuration interaction. Our results suggest that hydrated dielectrons are thermodynamically unstable relative to separated (single) hydrated electrons, although we also show that increasing the pressure could drive the equilibrium toward the formation of dielectrons. Because the simulations suggest that hydrated dielectrons are kinetically stable, we also examine a scenario for creating metstable, nonequilibrium populations of dielectrons, which involves the capture of a newly injected electron by a preexisting, equilibrated hydrated electron. These calculations, which allow for the full nonadiabatic relaxation of the injected electron, show that hydrated electrons may indeed act as trapping sites for unequilibrated electrons, so that capture may be a viable mechanism for creating dielectrons. We suggest possible experimental procedures to create such nonequilibrium hydrated dielectrons using either pulse radiolysis or ultrafast spectroscopic techniques.
\end{abstract}

\section{Introduction}

The hydrated electron is a unique chemical species formed by injecting an excess electron into liquid water by pulse radiolysis, ${ }^{1}$ by multiphoton ionization, ${ }^{2}$ or by electron photodetachment of a solute (e.g., the CTTS excitation of iodide) ${ }^{3}$ The most common view is that the hydrated electron is a solventsupported state that occupies a nearly spherical cavity with six water molecules in the first solvation shell, ${ }^{4}$ a picture supported by the results of computer simulations. ${ }^{5,6}$ Continuum dielectric calculations, ${ }^{7}$ which are consistent with the more recent simulation results, suggest that the hydrated electron may be viewed as a polaron, that is, as an electronic state stabilized by the polarization of the surrounding solvent.

Continuum dielectric calculations also suggest that if the solvent dielectric constant is large enough, as in water or ammonia, the energy of two electrons confined to a single cavity could be less than that of two (single) electrons, implying that solvated dielectrons, or bipolarons, should be stable. ${ }^{8}$ In fact, there is indirect experimental evidence for the formation of bound pairs of electrons in solutions of metals dissolved in liquid ammonia; the metal/ammonia solutions show a decrease in magnetic susceptibility at high concentrations of excess electrons, suggesting that the electrons' spins become paired. ${ }^{9}$ Mixed quantum/classical density functional calculations also have suggested that bound pairs of electrons form at high electron densities in liquid ammonia. ${ }^{10}$ In water, simulations have shown ${ }^{11}$ that hydrated dielectrons occupy a larger, less spherical cavity than (single) hydrated electrons. In contrast to the ammonia simulations, where only singlet-paired electrons were found to form dielectrons, simulations in water suggest that both singlet and triplet hydrated dielectrons can form, and that the dielectron's shape depends on the total spin. ${ }^{12,13}$

To date, there has been no unambiguous experimental confirmation of the existence of hydrated dielectrons. The existence of hydrated dielectrons has been reported in pulse radiolysis experiments, ${ }^{14}$ but this identification has been challenged and the signals reported as characteristic of dielectrons have been viewed by some as artifacts. ${ }^{15}$ Interest in hydrated dielectrons also has been stirred by the more recent pulse radiolysis experiments of Schmidt and Bartels, ${ }^{16}$ which explained the lack of an ionic strength effect in the bimolecular annihilation of hydrated electrons by the formation of pairs of electrons that subsequently react with two water molecules to evolve molecular hydrogen. Inspired by these experiments and emboldened by recent theoretical and computer advances, we have elected to compute the thermodynamic stability of dielectrons from simulation, calculating not just the energy but the free energy of hydrated dielectrons, which includes the entropy of solvation. In this paper, we present calculations of the free energies of the (single) hydrated electron and the singlet hydrated dielectron from mixed quantum/classical computer simulation; we chose not to calculate the free energy of the triplet hydrated dielectron because singlet dielectrons are energetically more stable than triplet dielectrons by tens of $k_{\mathrm{B}} T,{ }^{13}$ 
and thus singlet dielectrons are more likely to be stable even once entropy is taken into account.

Our approach to calculating the excess free energy of hydrated dielectrons relative to two separated (single) hydrated electrons is based on thermodynamic integration (TI). ${ }^{17} \mathrm{We}$ use TI to compute the differences in free energy among simulation cells containing classical water and either zero, one, or two excess electrons (with the two excess electrons bound in a single cavity as a singlet dielectron). As far as we are aware, the results presented here represent the first calculations of the thermodynamic properties of an explicitly quantum mechanical hydrated solute from mixed quantum/classical simulation and TI. Because TI allows the calculation of Helmholtz free energies and the internal energies are already known from simulation, ${ }^{13}$ we also are able to calculate the entropy differences between the different solvent-supported species. In addition, we use thermodynamic identities to calculate the Gibbs free energy differences among the zero, one, and two excess electron simulations, which allows us to examine the stability of hydrated dielectrons as a function of applied pressure.

The rest of the paper is organized as follows. Section II describes the simulation methods we use to calculate the thermodynamics of hydrated electrons and dielectrons. The excess electrons are treated quantum mechanically and all thermodynamic averages are computed from adiabatic molecular dynamics simulations, which are based on a full configuration interaction $(\mathrm{CI})$ calculation of the two-electron adiabatic eigenstates. ${ }^{13}$ In Section III, we review the technique of TI and compute the Helmholtz free energies of simulated water with zero, one, and two excess electrons. We then use thermodynamic identities to correct for the changes in pressure caused by the excluded-volume and electrostriction effects that occur when electrons are added to water in a fixed-volume simulation. We find that hydrated dielectrons are not thermodynamically stable with respect to dissociation into two separate (single) hydrated electrons. We have shown previously, however, that once formed, hydrated dielectrons are kinetically stable on time scales longer than tens of picoseconds (the longest times over which they have been simulated). ${ }^{13}$ This kinetic stability suggests that under nonequilibrium conditions, hydrated dielectrons could exist long enough for the chemical processes described by Schmidt and Bartels to take place. ${ }^{16}$ Thus, in Section IV we describe the possibility of making a nonequilibrium population of injected hydrated electrons that may be captured by preexisting equilibrated hydrated electrons to form metastable hydrated dielectrons. We simulate this capture process by injecting a second electron into one of the high-lying adiabatic two-electron eigenstates of a box containing a (single) hydrated electron, and calculating the nonadiabatic relaxation of the two electrons to their ground state. ${ }^{12} \mathrm{We}$ find that as long as the second electron is confined to be within a simulation-cell length (18 $\AA$ ) of the preexisting electron, the preexisting hydrated electron captures the injected electron $\sim 35 \%$ of the time to form a kinetically stable dielectron. This suggests that pulse radiolysis production of hydrated electrons may indeed create large populations of dielectrons, so that our prediction that dielectrons are thermodynamically unstable is not inconsistent with Schmidt and Bartels' results. ${ }^{16}$ Finally, we conclude in Section V with a discussion of the significance of our results and a description of possible experiments that may verify the production of hydrated dielectrons via nonequilibrium capture.

\section{Computational Methods}

Our calculations of the thermodynamic stability of hydrated electrons and dielectrons are based on mixed quantum/classical
(QM/CM) molecular dynamics simulations that treat the water molecules classically and the excess electron(s) quantum mechanically. We have described our approach to such QM/ CM simulations in detail elsewhere, ${ }^{12,13}$ so we give only a short summary here. The simulations take place in a cubic box 18.17 $\AA$ on a side containing 200 classical, flexible water molecules that interact through SPC-flex potentials ${ }^{18}$ and zero, one, or two quantum mechanical electrons that interact with the water molecules via a pseudopotential derived by Schnitker and Rossky. ${ }^{19}$ Other pseudopotentials have been developed that produce better agreement with the experimental spectrum of hydrated electrons, ${ }^{20}$ but for the purposes of comparison to previous work, we chose to use Schnitker and Rossky's pseudopotential. ${ }^{21}$ Minimum-image periodic boundary conditions ${ }^{22}$ were used for the classical solvent-solvent interactions, and all of the interactions were smoothly tapered to zero at half the box length. ${ }^{23}$ The positions and velocities of the classical water molecules were propagated by using the velocity Verlet algorithm, ${ }^{22}$ with a time step of $1 \mathrm{fs}$.

The QM part of the problem is solved by using a similar approach for both one and two excess electrons. For one excess electron, at each solvent configuration we use an iterative and block-Lanczos procedure to find the ground adiabatic eigenstate on a $16 \times 16 \times 16$ cubic grid. ${ }^{24}$ The charge density of this ground state is used to determine the force the electron exerts on the classical solvent molecules according to the HellmannFeynman formula. For two excess electrons, whose spins we take to be singlet paired, we use the two-electron charge density to compute the Hellmann-Feynman force. For each solvent configuration, we find the two-electron charge density by first solving for the lowest 10 adiabatic single-electron eigenstates on a $16^{3}$ grid as described above, and then constructing the twoelectron eigenstates using configuration interaction (CI) as described in ref 12 . Because the electrons are singlet paired, the CI calculations use a two-electron product basis constructed from all spatially symmetric combinations of the lowest 10 single-electron eigenstates, for a total of 55 product basis states. We compute the Coulomb and exchange energies in this basis with an efficient real-space quadrature. ${ }^{12}$ To further speed up the CI calculations, we also make use of what we have called the important states approximation, ${ }^{12}$ in which the two-electron problem is solved by using a restricted set of product-basis states and the full CI matrix is constructed only infrequently (in this case, every $3 \mathrm{fs}$ ) to determine the best restricted basis set to use. The full CI two-electron molecular dynamics calculations reported here take approximately 3 days of computational effort per picosecond of simulated dynamics on a single-processor AMD Athlon XP 1700 workstation.

For the nonequilibrium, nonadiabatic simulations of electron capture described in Section IV, we used the mean-field with surface hopping (MF/SH) algorithm of Prezhdo and Rossky. ${ }^{25}$ Our particular modification of the algorithm for use with two quantum particles is described in ref 12; further details of how we perform nonequilibrium, nonadiabatic full CI simulations of dielectrons can be found in ref 26 .

\section{The Thermodynamics of Hydrated Electrons and Dielectrons}

In previous work, we calculated the average energies of singlet dielectrons and (single) hydrated electrons from mixed quantum/classical simulations; the values we obtained are summarized in Table 1 . The ca. $-6 \mathrm{eV}$ energy of the spin singlet dielectron is fully $\sim 0.5 \mathrm{eV}$ lower than the energy of two infinitely separated electrons, so that energetically, the singlet 
TABLE 1: Calculated Thermodynamic Parameters for Neat (SPC/Flex) Water, Water with a Single Hydrated Electron, and Water with a Spin-Singlet Dielectron ${ }^{29, a}$

\begin{tabular}{lccc}
\hline & neat water $(\mathrm{eV})$ & electron $(i=1)$ & dielectron $(i=2)$ \\
\hline$E_{\mathrm{QM}}(\mathrm{eV})$ & & $-2.74(0.14)$ & $-5.99(0.17)$ \\
$V_{\mathrm{aq}}(\mathrm{eV})$ & $-88.64(0.17)$ & $-87.06(0.17)$ & $-82.38(0.56)$ \\
$E_{\text {int }}(\mathrm{eV})$ & $-88.64(0.17)$ & $-89.80(0.22)$ & $-88.37(0.59)$ \\
$A_{i}(N, V, T)-A_{i-1}(N, V, T)(\mathrm{eV})$ & & $-0.38(0.09)$ & $1.25(0.13)$ \\
$T\left[S_{i}(N, V, T)-S_{i-1}(N, V, T)\right](\mathrm{eV})$ & $-1.19(0.12)$ & $-0.78(0.29)$ & $-0.18(0.64)$ \\
$p^{\mathrm{CL}} i V(\mathrm{eV})$ & & $2.59(0.37)$ & $8.27(0.96)$ \\
$p^{\mathrm{QM}}{ }_{i} V(\mathrm{eV})$ & $-282(35)$ & $-3.71(0.04)$ & $-11.68(0.21)$ \\
$p_{i}(\mathrm{~atm})$ & & $-298(95)$ & $-898(253)$ \\
$G_{i}\left(N, p_{0}, T\right)-G_{i-1}\left(N, p_{0}, T\right)(\mathrm{eV})$ & & $-0.38(0.09)$ & $1.22(0.13)$
\end{tabular}

${ }^{a}$ The two standard deviation errors are given in Parentheses. In the conversion to $p_{0}$, we use the experimental compressibility of liquid water, not the (smaller) compressibility found in our simulations (see discussion in section III.B)

hydrated dielectron would appear to be stable. This is precisely the reasoning used in the continuum calculations that predicted dielectrons, ${ }^{8}$ but this reasoning is not valid because it does not take into account the energetic changes in the interactions between water molecules that occur when the water rearranges to solvate the excess charge. For example, Table 1 shows that although the quantum energy of the (single) hydrated electron is $\sim 2.7 \mathrm{eV}$ below the gas-phase continuum energy, the total internal energy of the system, $E_{\mathrm{int}}=E_{\mathrm{QM}}+V_{\mathrm{aq}}$, is only $\sim 1.2$ $\mathrm{eV}$ less than neat water. Thus, it costs neat water $\sim 1.5 \mathrm{eV}$ to form a cavity and solvate the electron, but the favorable electron-water interactions more than make up the penalty. To perform a similar analysis for the hydrated dielectron, we must compare the total energy of a simulation containing only water plus the energy of one containing a hydrated dielectron to twice the energy of a simulation of water containing a (single) hydrated electron. The internal energies in Table 1 show that the dielectron/water system is $\sim 2.6 \mathrm{eV}$ higher in energy than two separated (single) hydrated electrons. Evidently, the water structure is broken so much in solvating a hydrated dielectron that the favorable solvation energy cannot make up the difference. Thus, despite the fact that the energy of the singlet dielectron's ground state is $\sim 0.5 \mathrm{eV}$ less than the ground state energy of two (single) hydrated electrons, we conclude that dielectrons should not be considered energetically stable.

In view of the significant energetic instability of singlet hydrated dielectrons, it is clear that the only way this species can be stable is if there is a large entropic increase associated with dielectron formation: In other words, the free energy of the dielectron must be less than that of two (single) hydrated electrons. Therefore, to fully examine the question of dielectron stability, we must calculate the entropy difference between two (single) hydrated electrons and a hydrated dielectron. With the finite size of our simulation cell it does not make sense to examine stability by comparing the free energy of a dielectron in a single simulation cell to that of two separated electrons in the same small cell: In such a calculation, a significant fraction of the free energy could end up coming from nontrivial interactions between the water molecules in the first solvation shells of the separate hydrated electrons. We choose, therefore, to calculate the free energy of 200 water molecules at a standard temperature and density, the free energy of the same 200 water molecule simulation cell with a single excess electron, and the free energy of the cell with two excess electrons bound together in the form of a dielectron. We will then determine thermodynamic stability by comparing the free energies of two pairs of simulation boxes: one pair with a single electron in each box, and the other pair with a dielectron in one box and only water in the other box. These simulation cell pairs are illustrated schematically in Figure 1.

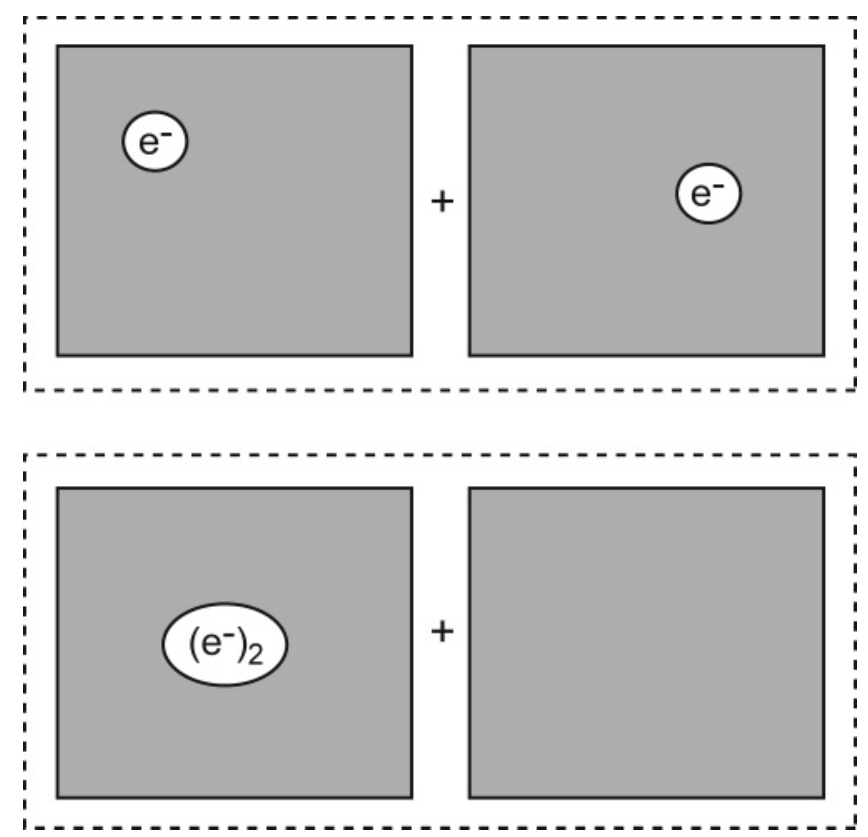

Figure 1. Schematic of the pairs of simulation boxes used to determine thermodynamic stability of hydrated dielectrons relative to two (single) hydrated electrons (cf. eq 1). The upper two boxes represent two separate simulation cells, each of which contains one excess electron. The lower two boxes represent two simulation cells, one of which contains two excess electrons in the form of a singlet dielectron, and the other of which contains only water.

We denote the Helmholtz free energy of a simulation cell consisting of water and $i$ excess electrons by $A_{i}$. The difference in free energy, $\Delta A$, between a dielectron and two separated electrons for the two-simulation-box scenario is

$$
\begin{aligned}
\Delta A=A_{2}+A_{0}-2 A_{1}-k_{\mathrm{B}} T \log 2 & =\left(A_{2}-A_{1}\right)- \\
& \left(A_{1}-A_{0}\right)-k_{\mathrm{B}} T \log 2
\end{aligned}
$$

where $k_{\mathrm{B}}$ and $T$ are Boltzmann's constant and the temperature, respectively, and the last term arises from the two ways of choosing which box will have the dielectron. The sign of $\Delta A$ in eq 1 determines the stability of the hydrated dielectron. The multiple box idea may be easily generalized to calculate the free energy of a system containing many electrons and dielectrons by envisioning $N$ boxes of water, with $M_{1}$ of these containing a (single) hydrated electron, $M_{2}$ containing a hydrated dielectron, and the remainder containing only water:

$$
\begin{aligned}
& A\left(N, M_{1}, M_{2}\right)=N A_{0}+M_{1}\left(A_{1}-A_{0}\right)+\mathrm{M}_{2}\left(A_{2}-A_{0}\right)+ \\
& k_{B} T\left[\log \left(N-M_{1}-M_{2}\right) !+\log M_{1} !+\log M_{2} !-\log N !\right]
\end{aligned}
$$


where the choice $A(2,0,1)-A(2,2,0)$ in eq 2 reproduces the free energy difference given in eq 1 . For a given concentration of electrons per simulation cell, $M_{1} / N$, one can find the concentration of dielectrons by minimizing the free energy with respect to $M_{2}$, leading to the usual formula for the equilibrium constant of a bimolecular reaction.

In the next subsection, we describe the theoretical methods used to calculate the Helmholtz free energy differences needed for use in eqs 1 and 2 . None of the calculations include contributions to the free energy from the spin of the electrons (spin is included only in the symmetrization requirement for the wave function of the singlet dielectron), so we add $-k_{\mathrm{B}} T$ $\log 2$ to the simulated free energy of the single-electron calculations to account for the two possible spin states of the unpaired (single) electron. The analogous change for many electrons would be to add $-M_{1} k_{\mathrm{B}} T \log 2$ to eq 2 .

A. Calculation of Helmholtz Free Energies from Thermodynamic Integration. Given a canonical partition function, $Z_{H}$, of a system described by a Hamiltonian $H$, the Helmholtz free energy, $A_{H}$, is

$$
A_{H}=-k_{\mathrm{B}} T \log Z_{H}
$$

where $Z_{H}=\operatorname{Tr}\left\{e^{-\beta H}\right\}$ and $\operatorname{Tr}$ denotes the trace operation, which sums and integrates over all possible values of all of the degrees of freedom. It is well-known that absolute free energies can be difficult to compute by simulation, but free energy differences may be found by using the trick of themodynamic integration (TI), also called "charging". ${ }^{17}$ In brief, TI finds the free energy difference between two systems, one described by a Hamiltonian $H_{0}$ and the other by a Hamiltonian $H_{0}+H^{\prime}$, by considering a fictitious Hamiltonian $H=H_{0}+\lambda H^{\prime} ; H^{\prime}$ is called the charging potential. By taking the derivative of the free energy with respect to $\lambda$, and using the fact that the operations all take place inside a trace so that factors such as $H_{0} H^{\prime} H_{0}$ can be reordered into the form $H^{\prime} H_{0} H_{0}$, one finds that $\mathrm{d} A_{H} / \mathrm{d} \lambda=\left\langle H^{\prime}\right\rangle_{H_{0}+\lambda H^{\prime}}$, where the brackets denote an equilibrium average and the subscript indicates the Hamiltonian used for the averaging. ${ }^{27}$ With the fundamental theorem of calculus, this implies that, ${ }^{17}$

$$
A_{H_{0}+H^{\prime}}-A_{H_{0}}=\int_{0}^{1} \mathrm{~d} \lambda\left\langle H^{\prime}\right\rangle_{H_{0}+\lambda H^{\prime}}
$$

Equation 4 shows that the difference in free energies between different systems can be found by smoothly changing one system into the other (we call each realization of such a change a "path") and calculating the average charging energy, $\left\langle H^{\prime}\right\rangle_{H}$, along the path in an equilibrium simulation. As mentioned above, our calculations do not explicitly include entropic factors due to spin, so at room temperature the free energy of an unpaired electron would be $k_{\mathrm{B}} T \log 2 \simeq 0.017 \mathrm{eV}$ less than the value found from charging; this correction, however, is much smaller than the statistical error of the simulations.

To find the free energy differences needed in eqs 1 and 2, we perform TI using the following pathways:

$$
\begin{gathered}
A_{1}-A_{0}: \quad H_{\mathrm{el}}(\lambda)=H_{w}+\lambda H_{1} \\
\tilde{A}_{2}-A_{1}: \quad H_{\text {diel }}(\lambda)=H_{w}+\left(H_{1}+H_{2}\right) / 2+\lambda\left(H_{1}+H_{2}\right) / 2
\end{gathered}
$$

$$
A_{2}-\tilde{A}_{2}: \quad H_{\text {diel }}(\lambda)=H_{w}+H_{1}+H_{2}+\lambda V_{12}
$$

where $H_{w}$ denotes the Hamiltonian for the classical degrees of freedom, $H_{i}$ denotes the Hamiltonian for excess electron $i$, including its interaction with the water molecules, and $V_{12}$

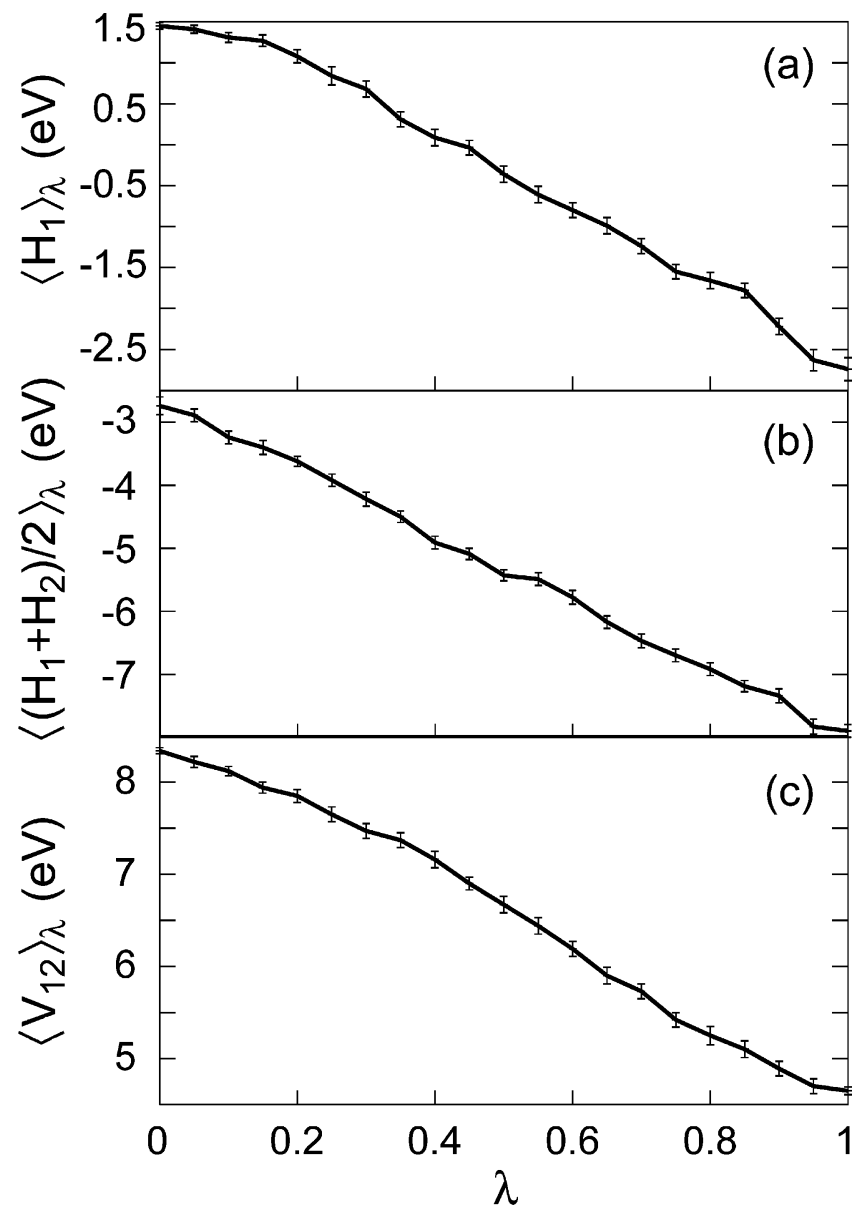

Figure 2. Average charging energies as functions of integration parameter $\lambda$ for thermodynamic integration along the pathways given by eqs $5 \mathrm{a}$ (panel a), $5 \mathrm{~b}$ (panel $\mathrm{b}$ ), and $5 \mathrm{c}$ (panel c). The integrals over $\lambda$ of these curves (eq 4) are reported in Table 1. The error bars shown are two standard deviations.

denotes the electron-electron interaction. The first pathway, eq 5a, takes us from a box containing only water molecules to one that also has a single excess electron. The second pathway, eq $5 b$, slowly adds a second electron but does not include the repulsion between the two electrons, and the third pathway, eq $5 \mathrm{c}$, turns on the interaction between the electrons. The charging pathway from one to two electrons was split into two legs because we found initially that charging along a single path,

$A_{2}-A_{1}: \quad H=H_{w}+\left(H_{1}+H_{2}\right) / 2+\lambda\left[\left(H_{1}+H_{2}\right) / 2+V_{12}\right]$

led to final configurations having two separate hydrated electrons in the simulation cell. Later tests revealed that the production of two (single) hydrated electrons was caused by a programming error that doubled the electron-electron repulsion. By the time this error was discovered, the charging calculations along the two legs had been completed. We also have run less extensive calculations using the pathway given by eq 6 and we find the same value for $A_{2}-A_{1}$ as obtained using eqs 5 to within the simulation error.

Figure 2 displays the charging energy as a function of $\lambda$ for each of the three charging pathways described by eqs $5 \mathrm{a}-\mathrm{c}$. Each point was generated by changing $\lambda$ and running with Hamiltonian $H(\lambda)$ until the system reached equilibrium, typically 1-4 ps. For each value of $\lambda$, the charging energy was then calculated over an additional 10 ps trajectory for the paths given by eqs $5 \mathrm{a}$ and $5 \mathrm{~b}$, and over an additional 3 to 6 ps trajectory 
for the path given by eq $5 \mathrm{c}$, and the average and standard deviation of the mean was computed. ${ }^{28}$ The average for the full dielectron ( $\lambda=1$, bottom panel of Figure 2) was taken from the 30-ps equilibrium simulation described in ref 13 , and the average single-electron value came from a 60-ps adiabatic simulation. The integrals over $\lambda$ required to calculate the free energy differences, eq 4 , were performed by using an extended Simpson's rule, and the results of integrating the data in Figure 2 (eq 4) are given in Table 1. The large positive value of $\Delta A=$ $1.6 \mathrm{eV}=A_{2}+A_{0}-2 A_{1}$ implies that singlet dielectrons are thermodynamically unstable.

B. Pressure Correction and Gibbs Free Energies. The Helmholtz free energy differences calculated above are not the desired quantities to predict stability for constant pressure experiments. Unfortunately, the volume of our simulation cells is not large enough for the pressure fluctuations in the isothermal, isochoric $(N, V, T)$ ensemble to be small enough that the results would be equivalent to those from the isothermal, isobaric $(N, p, T)$ ensemble. In view of the volume taken up by the electron and dielectron, it is possible that the pressure in the liquid changes by enough to shift the equilibrium at constant volume. Therefore, in this subsection, we change ensembles and calculate the stability of dielectrons at fixed pressure, effectively converting our Helmholtz free energies, $A_{i}$, to Gibbs free energies, $G_{i}$,

$$
G_{i}\left(N, p_{i}, T\right)=A_{i}(N, V, T)+p_{i} V
$$

where $p_{i}$ is the average pressure in the appropriate $(N, V, T)$ simulation; the way this pressure is calculated using the virial is described in the Appendix. Stability at a common pressure, $p$, is determined (cf. eq 1) by the sign of the Gibbs free energy difference,

$$
\Delta G=G_{2}(N, p, T)+G_{0}(N, p, T)-2 G_{1}(N, p, T)-k_{\mathrm{B}} T \log 2
$$

which requires the set of $G_{i}$ at distinct pressures $p_{i}$ to be converted into a set of $G_{i}$ for a common pressure, $p$. This conversion, which depends on both the simulated pressures and compressibilities of our system, is performed by using eq A3, as described in the Appendix.

The pressures for our neat water, single hydrated electron, and singlet dielectron boxes were computed from trajectories 2 $\mathrm{ns}, 200 \mathrm{ps}$, and $30 \mathrm{ps}$ long, respectively; the average pressure values are given in Table 1 and the pressure probability distributions are shown in Figure 3. Figure 3 and Table 1 make it clear that adding an excess electron to water causes an increase in pressure due to water-water interactions $\left(p_{1}^{\mathrm{CL}}\right)$ but a decrease in pressure due to electron-water interactions $\left(p_{1}^{\mathrm{QM}}\right)$, the result of electrostriction. Adding a second electron to form a dielectron further increases the pressure due to water-water interactions $\left(p_{2}^{\mathrm{CL}}\right)$, but the pressure decrease due to electrostriction increases greatly in magnitude, leading to a larger total pressure change on converting a (single) hydrated electron into a dielectron $(-600 \mathrm{~atm})$ than comes from adding a hydrated electron to neat water $(-18 \mathrm{~atm})$.

The pressure fluctuations shown in Figure 3 allow the calculation of the adiabatic compressibility, $\kappa_{\mathrm{S}}$, for SPC/Flex water; as discussed in the Appendix, we find that $\kappa_{\mathrm{S}}=0.7 \times$ $10^{-10} \mathrm{~m}^{2} / \mathrm{N}$, roughly one-eighth the experimental compressibility. This implies that finite size effects on the pressure in our simulations should be smaller than those in models having more realistic compressibilities. Equation A3 suggests that the correction to the Gibbs free energy difference will be small, so

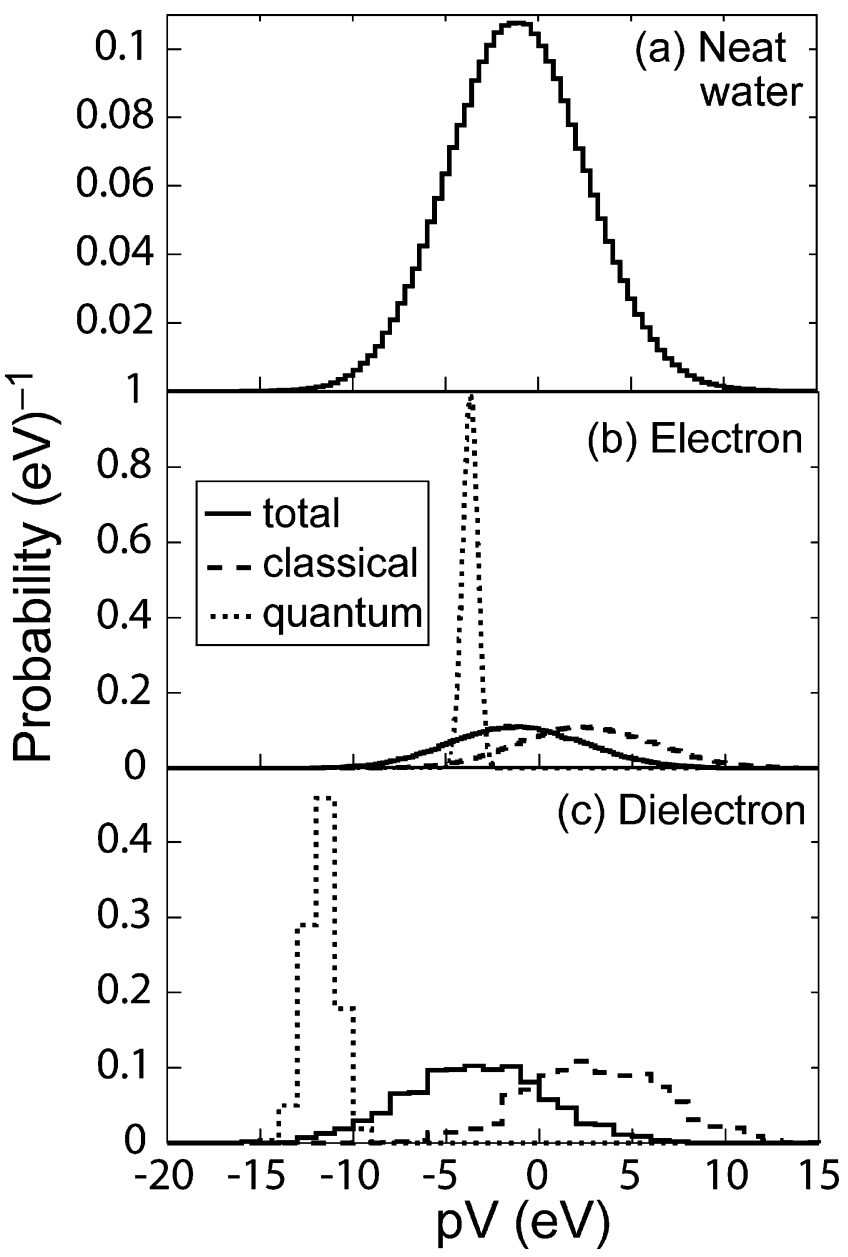

Figure 3. Distribution of pressures for neat (SPC/flex) water (panel a), water with a hydrated electron (panel b), and water with a singlet hydrated dielectron (panel c); the pressures are calculated by using both classical and quantum contributions from the virial (eq A1).

to maximize the possibility that the pressure correction will stabilize the dielectron, we use the experimental isothermal compressibility, $\kappa_{\mathrm{T}}=4.6 \times 10^{-10} \mathrm{~m}^{2} / \mathrm{N},{ }^{30}$ to correct the Gibbs free energy difference, $\Delta G$. Plugging the experimental compressibility and the calculated pressures into eq A3 gives a linear stability curve as a function of $\left(p-p_{0}\right)$ with a slope of $-9.9 \times$ $10^{-5} \mathrm{eV} / \mathrm{atm}$. The negative slope means that although separated (single) hydrated electrons have a much lower Gibbs free energy than a dielectron, increasing the pressure can drive the equilibrium toward dielectron formation, in accord with Le Chatelier's principle. The small magnitude of the slope, however, tells us that for near-ambient pressures, the pressure corrections are small and thus hydrated dielectrons are still expected to be thermodynamically unstable.

C. Discussion. Since the pressure corrections make little difference to the stability, the conclusion of our TI calculations seems clear: Dielectrons are thermodynamically unstable. What, then, is to be made of the experimental indications that they exist? ${ }^{16}$ One possibility is simply that our model is inadequate, possibly due to errors in the pseudopotential used for the electron-water interaction. We view this possibility as unlikely because the calculated free energy difference is so large that it is difficult to see how reasonable changes to the pseudopotential will be able to shift the energies or entropies by enough to make much difference. Another possibility is that although dielectrons are thermodynamically unstable, they might be kinetically stable if a nonequilibrium population of dielectrons was formed. We will examine how one might form such an athermal population 
in the next section. Finally, the species observed by Schmidt and Bartels may be a complex of two nearby (single) hydrated electrons, rather than a dielectron confined to a single cavity.

Using the total internal energies (classical potential plus quantum) of the different simulation boxes, one can compute the differences in entropy among the different systems, as shown in Table 1. We note that the entropy and free energy differences for hydrated electron solvation we report here cannot be compared directly to those reported experimentally, ${ }^{31}$ because we take the zeroes of free energy and entropy to be the state consisting of neat water, whereas the experiments take the zero to be that of the hydrated proton..$^{32}$ The negative free energy difference that accompanies addition of an electron to neat water is caused by the $1.16 \mathrm{eV}$ decrease in the internal energy of the system, although this decrease is compensated by the $0.78 \mathrm{eV}$ increase in free energy due to the smaller entropy of the electronplus-water system. Changing from one electron to two electrons entails no entropic cost within the error of our simulations. The instability of the singlet dielectron is therefore caused by the energy penalty paid by the water to solvate the larger, less spherical dielectron. The extra solvation allowed by having more waters in the first solvation shell makes the ground-state energy of the dielectron ca. $-6.0 \mathrm{eV}$, fully $0.5 \mathrm{eV}$ less than the ca. $-5.5 \mathrm{eV}$ of a two separated hydrated electrons, ${ }^{13}$ but this is more than compensated by the $3.1 \mathrm{eV}$ increase in the waterwater potential. Apparently, the attractive energy due to electrostriction is outweighed by the disruption of solvent structure caused by the larger volume of the dielectron, with entropy playing no significant role.

\section{Capture Mechanism for Hydrated Dielectron Formation}

Given that our calculations indicate that hydrated dielectrons are thermodynamically unstable relative to widely separated (single) hydrated electrons, forming hydrated dielectrons in the laboratory cannot be a matter of creating a large concentration of (single) hydrated electrons and waiting for an equilibrium population of dielectrons to form. In this section, we consider an alternative mechanism that would allow creation of a nonequilibrium, metastable population of dielectrons. Our idea is that a (single) hydrated electron provides an attractive well that can capture an additional electron if a nonequilibrium (free) electron were in the vicinity. In the absence of electron-electron repulsion, the polarization of the water surrounding a preexisting hydrated electron is highly attractive for a free electron, so the question is whether this attraction is enough to overcome the repulsion between electrons. It is straightforward to calculate the repulsion between two electrons in a (single) hydrated electron cavity, but such a calculation would not include the extra stabilization caused by changes in the size and shape of the cavity upon forming the dielectron. Thus, in this section, we study the capture of an excess electron by a preexisting (single) hydrated electron. We do this by modeling the injection of additional electrons into a preexisting population of hydrated electrons, including both the nonadiabatic relaxation of the two electrons and the relaxation of the solvent. Experimentally, one might imagine a pulse radiolysis experiment producing just such a situation: Electrons produced by the leading edge of the pulse may have equilibrated by the time additional electrons are produced by the trailing edge of the pulse. One also could envision creating a population of electrons by using an ultrafast laser pulse and then creating more electrons with a second pulse a few picoseconds later, after the electrons from the first pulse had equilibrated.

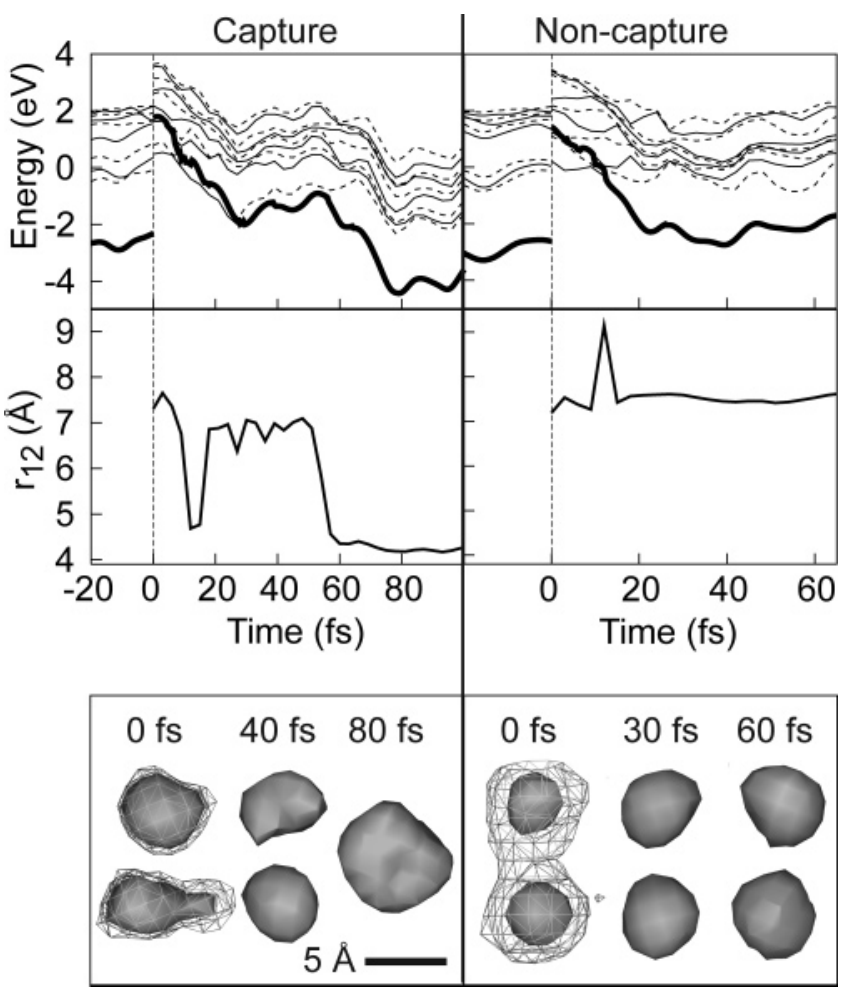

Figure 4. Dynamical histories of two nonequilibrium injection runs, in which a second electron is added at time zero to a simulation cell that is already equilibrated for a single excess electron; the two-electron system initially is taken to be in the fourth adiabatic eigenstate, as discussed in Section IV. The panels on the left are for a run in which the second electron is captured by the first, whereas the panels on the right are for a run in which the second electron formed a new hydrated electron. The upper panels display the dynamical histories of the quantum mechanical energy levels, where the alternating thin solid and dashed curves are the adiabatic energies and the thick solid curves represent the energy of the mean-field wave function (see refs 12 and 25 for details). Before time $t=0 \mathrm{fs}$, the energies are for a single excess electron, and at later times, the energies are the two-electron energies calculated with full CI. The middle panels show the root-mean-square electron-electron separation for the occupied adiabatic eigenstate, $\left\langle\psi\left|\left(\hat{r}_{1}-\hat{r}_{2}\right)^{2}\right| \psi\right\rangle^{1 / 2}$, following the injection. The bottom panels show isodensity contours of the occupied two-electron adiabatic eigenstate for the times indicated; the contours are taken to be at $10 \%$ of the maximum charge density for each case; the mesh plots at time zero indicate isodensity countours at $5 \%$ of the maximum density to show the delocalized nature of the injected electron.

Our modeling of this hypothetical capture process starts by taking equilibrated (single) hydrated electron configurations and adding a second electron to the box in an excited state; we take the initial electronic configuration to be the fourth dielectronic state, because this state consists largely of configurations having one electron in the ground single-electron state and the other electron in several of the delocalized single-electron states from the continuum. After the injection, the two-electron system, which was constrained to be spin singlet at all times, was allowed to relax nonadiabatically to equilibrium. ${ }^{33}$ In seven out of twenty runs the two electrons relaxed to form a dielectron, and in the other runs the system formed two separated hydrated electrons.

Figure 4 displays the results of two of the injection runs; the panels on the left show a trajectory in which the injected electron was captured by the preexisting electron to form a dielectron, whereas the panels on the right show one of the trajectories in which the injected electron was not captured. The upper left panel shows the dynamical history of the adiabatic energy levels (depicted as alternating solid and dashed curves with the 
occupied state shown as the heavy black solid curve) for the trajectory in which the injected electron was captured. Before $t=0$, the system has the electronic structure of a single hydrated electron, with an s-like ground state near $-3 \mathrm{eV}$ and three quasidegenerate $\mathrm{p}$-like excited states near $-0.5 \mathrm{eV}$. The second electron was added to the simulation cell at $t=0$ with the system placed in the fourth dielectronic state, and within $30 \mathrm{fs}$, the system reached the ground state after several nonadiabatic transitions. The ground state energy rapidly approaches ca. -4 $\mathrm{eV}$ and over a few hundred femtoseconds relaxes to ca. -6 $\mathrm{eV}$, which is the energy typical of the equilibrated singlet hydrated dielectron. For the same trajectory, the center left panel shows the value of the root-mean-square interelectron separation, $r_{12}=\left\langle\psi\left|\left(\hat{r}_{1}-\hat{r}_{2}\right)^{2}\right| \psi\right\rangle^{1 / 2},{ }^{13}$ where $\psi$ is the two-electron wave function of the $\mathrm{MF} / \mathrm{SH}$ reference state. ${ }^{25}$ Of course, there is no interelectron separation before time zero when there is only one electron in the box, but once the electron is injected, it is clear that the two electrons work to avoid each other, keeping their separation close to half the box size. The large fluctuations in $r_{12}$ are the result of the nonadiabatic transitions between states. By the time the system has reached the ground state, the interelectron separation is $r_{12} \sim 4 \AA$, which is also characteristic of the equilibrium singlet hydrated dielectron. ${ }^{13}$ The charge densities displayed in the panel on the bottom left also show that the initially delocalized two-electron system relaxes to form a more compact aspherical object inside a single cavity, i.e., a dielectron. This trajectory is typical of those that show capture, and these seven runs suggest that dielectrons can be formed within $\sim 300 \mathrm{fs}$ of injection if there is a preexisting equilibrated electron nearby.

The panels on the right side of Figure 4 show the same information as those on the left side of the figure, but for a trajectory in which the injected electron was not captured. The upper right panel shows that again the system takes $\sim 15$ fs to reach the ground state via nonadiabatic relaxation, but for this trajectory, once the system reaches the ground state, the adiabatic energies are much higher than those seen when the injected electron is captured. Not only are the adiabatic energies in this trajectory higher than those expected for an equilibrium singlet dielectron, they also are higher than what would be expected for two separated (single) hydrated electrons (ca. $-5.5 \mathrm{eV}$ for two separated electrons each in their ground state). However, the two electrons are still relatively close together (only $\sim 7 \AA$ apart), resulting in $\sim 2 \mathrm{eV}$ of Coulomb repulsion between them. If this Coulomb repulsion is subtracted off, the resulting energies are indeed only slightly higher than one would expect for two independent (single) hydrated electrons (the difference is likely due to heating resulting from the $\sim 3 \mathrm{eV}$ of energy added by injecting the second electron). We note that the long-time interelectron separation of $r_{12} \sim 7 \AA$ visible in the center-right panel is not unique: Other runs that yielded two separate hydrated electrons had the second electron form as far away as $r_{12} \sim 10 \AA$. Finally, the lower right panel shows that after relaxation, there are now two independent hydrated electrons in separated cavities in the box; the fact that the electrons are separate is verified by the fact that the calculated exchange energy of the two-electron ground state is very nearly zero.

These calculations strongly suggest that hydrated electron cavities can capture an additional electron. However, we do not know how to relate the simulated $35 \%$ capture probability to a rigorous capture cross section; this result, therefore, is only of a qualitative nature. It seems certain that limits on the cross section could be set by Golden Rule-type calculations of the rate of scattering of plane waves into the bound states of a single-electron-containing cavity, but such calculations would probably underestimate the role of solvent relaxation in allowing the capture. On the other hand, the simulations described in this section may overestimate the effectiveness of solvent relaxation in capture because the injected electron is always forced to stay within $9 \AA$ of the equilibrated electron due to the finite size of the simulation box. Within our current computational approach, we can conclude that putting an extra electron into water near a hydrated electron and keeping it there clearly favors the formation of kinetically stable dielectrons. It is not unreasonable, therefore, to believe that pulse radiolysis or multipulse multiphoton ionization experiments might lead to the formation of dielectrons in quantities not allowed by thermodynamic equilibrium.

\section{Concluding Remarks: The Thermodynamics of Hydrated Electrons and Dielectrons}

In this paper, we used mixed quantum/classical molecular dynamics simulations, combined with thermodynamic integration, to compute the thermodynamic stability of hydrated dielectrons relative to (single) hydrated electrons. To the best of our knowledge, this work represents the first theoretical calculations of the thermodynamics of either hydrated electrons or dielectrons. We found that hydrated electrons are thermodynamically stable by $\sim 0.4 \mathrm{eV}$ relative to a system consisting of neat water plus a zero-energy free electron; the stabilization comes from the large energetic benefit of solvating the electron, which is compensated somewhat by the reduced entropy of the solvent. We also found that hydrated dielectrons are thermodynamically unstable because the disruption of the solvent structure required to solvate a dielectron increases the solventsolvent interaction energy by significantly more than it costs to carve out two separate cavities. The energy penalty to the solvent more than makes up for the fact that the dielectron energy is $\sim 0.5 \mathrm{eV}$ less than the energy of two separated (single) hydrated electrons. In addition, our calculations showed that there is little additional entropic cost to solvate the dielectron: Most of the entropy change takes place when the first electron is solvated, and there is little entropic cost to further disrupting the solvent upon addition of the second electron.

Our calculated thermodynamic instability of dielectrons is not necessarily at odds with Schmidt and Bartels' observation ${ }^{16}$ that the presence of dielectrons could explain the lack of an ionic strength effect in the annihilation reaction of (single) hydrated electrons. In fact, Schmidt and Bartels concluded that two (single) hydrated electrons may form a stable, bound complex when their centers are $\sim 9 \AA$ apart, and that such complexes could produce dielectrons via tunneling of one electron into the other hydrated electron's "solvent trap". ${ }^{16}$ Although we have not addressed the stability of nearby single hydrated electron complexes, our calculations have demonstrated that a nearly free electron may be captured by a preexisting hydrated electron, so the tunneling mechanism suggested by Schmidt and Bartels seems plausible.

Because of the difficulty in modeling a realistic capture scenario in a small simulation cell, the calculations described in Section IV do not show definitively that an equilibrated hydrated electron can capture an additional electron. However, the simulations certainly suggest that (single) hydrated electrons could play this role. If this is the case, one would expect to be able to produce dielectrons in ways other than pulse radiolysis. For instance, one could use a sequence of two laser pulses to first create hydrated electrons by photoionization and then create additional electrons to be captured by the initial hydrated- 
electron population. We have suggested elsewhere ${ }^{26}$ that two additional laser pulses would give a unique pump-probe signature for dielectrons even in the presence of a large number of (single) hydrated electrons, so one could imagine a fourpulse optical experiment where the four pulses are used to (1) produce electrons, (2) produce electrons/dielectrons, (3) pump electrons/dielectrons, and (4) probe electrons/dielectrons. Such an experiment would not be easy, but it does, in principle, provide a method to produce dielectrons and observe them directly. One also could imagine identifying dielectrons by performing pump-probe spectroscopy on a sample prepared with pulse radiolysis, although with pulse radiolysis the pumpprobe signature of the dielectron may be obscured by the production of ions, radicals, and other species.

Our equilibrium simulations of dielectrons ${ }^{13}$ showed that both singlet-paired and triplet-paired dielectrons are stable to dissociation on time scales of the order of tens of picoseconds, or longer. In view of the predicted thermodynamic instability of dielectrons, it is clear that the kinetic stability will be of considerable interest if indeed dielectrons may be formed by capture. In particular, one would like to understand the rate of dielectron dissociation to compare it to the expected rates of the chemical reactions with the solvent that ultimately destroy the dielectrons. It is well-known that long equilibrium simulations will rarely produce dissociation events, but such events are often understood by forming the potential of mean force (PMF) for the reaction coordinate. We therefore should be able to understand the kinetic stability of dielectrons by computing the PMF between two excess electrons in liquid water as a function of electron-electron separation. The PMF should also shed light on the complexes of (single) hydrated electrons postulated by Schmidt and Bartels. ${ }^{16}$ To efficiently simulate configurations at many interelectron separations requires umbrella sampling $;{ }^{34}$ however, neither traditional umbrella sampling nor so-called quantum umbrella sampling ${ }^{35}$ can be used to separate two electrons in a controlled fashion. ${ }^{36}$ We recently have developed a new method for quantum umbrella sampling, so we defer calculation of the electron-electron PMF to a subsequent paper. $^{37}$

In summary, we have used thermodynamic integration with mixed quantum/classical molecular dynamics to study the thermodynamic stability of hydrated electrons and dielectrons. We found that hydrated electrons are thermodynamically favorable relative to free electrons and neat water, but that there is a significant entropic penalty paid by the solvent to accommodate a single excess electron. Our full CI calculations of dielectron stability showed that although there is little entropic cost on going from a (single) hydrated electron to a dielectron, the energy penalty due to disrupting the solvent structure makes dielectrons thermodynamically unstable. We also explored the possibility that the small size of our simulation box made dielectrons less stable due to changes in the pressure of the simulation caused by excluded volume and electrostriction effects, and found that the changes in pressure were too small to make dielectrons thermodynamically stable to dissociation. Finally, we explored the idea that (single) hydrated electrons can act as trapping centers for additional electrons, and we concluded that a (single) hydrated electron can capture an electron to form dielectrons. Thus, although the calculations presented in this paper predict that hydrated dielectrons are unstable, they also suggest possible experiments to produce nonequilibrium populations of kinetically stable dielectrons.

Acknowledgment. This work was supported by the NSF under Grant No. CHE-0204776. R.E.L. was a California
Nanosystems Institute/Hewlett-Packard Postdoctoral Fellow. B.J.S. is a Camille Dreyfus Teacher-Scholar. The charge densities shown in Figure 4 were produced with the UCSF Chimera package from the Computer Graphics Laboratory, University of California, San Francisco (supported by NIH P41 RR-01081). ${ }^{41}$ We gratefully acknowledge UCLA's Academic Technology Services for the use of its Hoffman Beowulf Cluster.

\section{Appendix A: Calculation of Pressures and Gibbs Free Energies}

To convert the Helmholtz free energies computed with TI into Gibbs free energies, we must first compute the average pressure in each of the simulation boxes. We calculated the pressure for the neat water, single electron, and singlet dielectron boxes from the virial, ${ }^{22}$

$$
p V=N k_{\mathrm{B}} T+\frac{1}{3} \sum_{j=1}^{N} \boldsymbol{F}_{j} \boldsymbol{r}_{j}
$$

where the sum runs over all the classical atoms. Since we are not simulating a constant temperature ensemble, we tried using both the instantaneous temperature and the average temperature in eq A1, and found that the pressure and its fluctuations were the same in both cases. The force on each water molecule is the sum of the classical forces from the other water molecules and the Hellmann-Feyman force from the electron(s). ${ }^{38} \mathrm{We}$ found it instructive to divide the pressure into a contribution from ideal-gas-plus-classical terms, $p^{\mathrm{CL}} V=N k_{\mathrm{B}} T+\sum_{j} \boldsymbol{F}_{j}^{\mathrm{CL}} \cdot \boldsymbol{r}_{j}$, and a contribution from the quantum forces, $p^{\mathrm{QM}} V=\sum_{j} \boldsymbol{F}_{j}^{\mathrm{QM}} \cdot \boldsymbol{r}_{j}$, so that the total pressure $p=p^{\mathrm{CL}}+p^{\mathrm{QM}}$.

We note that the simulated pressure depends on the details of how the long-range Coulomb interactions are treated with periodic boundary conditions. In the results reported here, we truncate the interactions at half the box length using a smooth cutoff. $^{23}$ The negative pressure we obtain means that absolute pressures cannot be meaningful for the density and temperature simulated; however, pressure differences should be meaningful provided the compressibility of SPC/Flex water is not dramatically different from that of real liquid water, as discussed below.

According to eq 8 , the stability of dielectrons at a single, common pressure, $p$, requires the Gibbs free energy differences among zero, one, and two excess electron simulations at that pressure. The calculated pressures for each simulation are different, however, so the Gibbs free energies at the common pressure must be determined by using the simulated free energies at distinct pressures. This is accomplished by writing each Gibbs free energy at pressure $p, G_{i}(N, p, T)$, in terms of the simulated Gibbs free energy, $G_{i}\left(N, p_{i}, T\right)$, using a Taylor series expansion in the pressure,

$$
\begin{aligned}
& G_{i}(N, p, T)=G_{i}\left(N, p_{i}, T\right)+ \\
& \quad\left(\frac{\partial G_{i}\left(N, p_{i}, T\right)}{\partial p}\right)_{N, T}\left(p-p_{i}\right)+\frac{1}{2}\left(\frac{\partial^{2} G_{i}}{\partial p^{2}}\right)_{N, T}\left(p-p_{\mathrm{i}}\right)^{2}+\ldots
\end{aligned}
$$

Because $\partial G / \partial p=V$ and $\partial^{2} G / \partial p^{2}=-V \kappa_{T}$, where $V$ is the system volume and $\kappa_{\mathrm{T}}$ is the isothermal compressibility, we find,

$$
\begin{gathered}
G_{2}(N, p, T)+G_{0}(N, p, T)-2 G_{1}(N, p, T) \simeq \\
A_{2}(N, V, T)+A_{0}(N, V, T)-2 A_{1}(N, V, T)- \\
\quad \frac{1}{2} V \kappa_{T}\left[\left(p-p_{2}\right)^{2}+\left(p-p_{0}\right)^{2}-2\left(p-p_{1}\right)^{2}\right]
\end{gathered}
$$

Note that eq $\mathrm{A} 3$ predicts a linear variation of $\Delta G$ with pressure 
even though we have computed the individual Gibbs free energies to second order in the pressure differences.

For simplicity in writing eq $\mathrm{A} 3$, we assumed that the isothermal compressibility is the same for each system simulated in this work. We have tested this assumption by using the fluctuations in the pressures simulated with zero-, one-, and twoexcess electrons to determine the adiabatic compressibility in each case. At room temperature, the adiabatic and isothermal compressibilities of liquid water are nearly the same, so we believe comparing adiabatic compressibilities is sufficient; more accuracy could be obtained by using the thermodynamic identity relating the isothermal and adiabatic compressibilities via the coefficient of thermal expansion and the specific heat, ${ }^{39}$ but we have not pursued this possibility. The mean-square fluctuations in the pressure give the adiabatic compressibility, $\kappa_{S},{ }^{40}$

$$
V\left\langle(p-\langle p\rangle)^{2}\right\rangle=k_{\mathrm{B}} T / \kappa_{\mathrm{S}}
$$

where $T$ is the average temperature. The same simulations that were used to compute the average pressure give $\kappa_{\mathrm{S}} \simeq 0.7 \times$ $10^{-10} \mathrm{~m}^{2} / \mathrm{N}$ for all three cases: a box containing 200 water molecules $\left((0.70 \pm 0.01) \times 10^{-10} \mathrm{~m}^{2} / \mathrm{N}\right)$; a box of 200 waters with one excess electron $\left((0.71 \pm 0.03) \times 10^{-10} \mathrm{~m}^{2} / \mathrm{N}\right)$; and a box containing 200 waters with a dielectron $((0.66 \pm 0.09) \times$ $\left.10^{-10} \mathrm{~m}^{2} / \mathrm{N}\right)$. The uncertainties in the aforementioned compressibilities were estimated by fitting Gaussians to the distributions of pressures and taking the uncertainties as those estimated by the curve fitting routine in Origin 7.0. To test for finite size effects, a 200-ps trajectory was run for a box containing 499 water molecules at the same density and temperature. The value of the pressure differed from the 200 water molecule value (Table 1) due to the increased cutoff distance for the interactions, but the compressibility of the larger system was the same. We note that for the reasons explained in the text, the corrected Gibbs free energies presented in Table 1 used the experimental value of the compressibility rather than the simulated value.

\section{References and Notes}

(1) See, e.g.: Bartels, D. M.; Takahashi, K.; Cline, J. A.; Marin, T. W.; Jonah, C. D. J. Phys. Chem. A 2005, 109, 1299.

(2) See, e.g.: Shi, X. L.; Long, F. H.; Lu, H.; Eisenthal, K. B. J. Phys. Chem. 1996, 100, 11903.

(3) See, e.g.: Vilchiz, V. H.; Kloepfer, J. A.; Germaine, A. C.; Lenchenkov, V. A.; Bradforth, S. E. J. Phys. Chem. A 2001, 105, 1711. (4) Kevan, L. Acc. Chem. Res. 1981, 14, 138.

(5) Schwartz, B. J.; Rossky, P. J. J. Phys. Chem. 1994, 98, 4489. Schwartz, B. J.; Rossky, P. J. J. Phys. Chem. 1995, 99, 2953. Schwartz, B. J.; Rossky, P. J. J. Chem. Phys. 1994, 101, 6917. Schwartz, B. J.; Rossky, P. J. Phys. Rev. Lett. 1994, 72, 3282. Schwartz, B. J.; Rossky, P. J. J. Mol. Liq. 1995, 65-6, 23. Schwartz, B. J.; Bittner, E. R.; Prezhdo, O. V.; Rossky, P. J. J. Chem. Phys. 1996, 104, 5942. Wong, K. F.; Rossky, P. J. J. Phys. Chem. A 2001 105, 2546. Wong, K. F.; Rossky, P. J. J. Chem. Phys. 2002, 116, 8418. Wong, K. F.; Rossky, P. J. J. Chem. Phys. 2002, 116, 8429.

(6) Neria E.; Nitzan, A.; Barnett, R. N.; Landman, U. Phys. Rev. Lett. 1991, 67, 1011

(7) Jortner, J. Mol. Phys. 1962, 5, 257. Jortner, J. J. Chem. Phys. 1959, 30, 839. Blandamer, M. J.; Catterall, R.; Shields, L.; Symons, M. C. R. J. Chem. Soc. 1964, 4357. Blandamer, M. J.; Shields, L.; Symons, M. C. R. J. Chem. Soc. 1965, 3759.

(8) Fueki, K. J. Chem. Phys. 1969, 50, 5381.

(9) Thompson, J. C. Electrons in Liquid Ammonia; Oxford University Press: London, UK, 1976.

(10) Deng Z. H.; Martyna, G. J.; Klein, M. L. Phys. Rev. Lett. 1992, 68, 2496. Deng Z. H.; Martyna, G. J.; Klein, M. L. Phys. Rev. Lett. 1993, 71, 267. Deng Z. H.; Martyna, G. J.; Klein, M. L. J. Chem. Phys. 1994, 100, 7590. Martyna, G. J.; Deng, Z. H.; Klein, M. L. J. Chem. Phys. 1993, 98,555 .

(11) Kaukonen, H.-P.; Barnett, R. N.; Landman, U. J. Chem. Phys. 1992 97, 1365.

(12) Larsen, R. E.; Schwartz, B. J. J. Chem. Phys. 2003, 119, 7672.

(13) Larsen, R. E.; Schwartz, B. J. J. Phys. Chem. B 2004, 108, 11760.
(14) Basco, N.; Kenney, G. A.; Walker, D. C. Chem. Commun. 1969, 917. Basco, N.; Kenney-Wallace, G. A.; Vidyarthi, S. K.; Walker, D. C. Can. J. Chem. 1972, 50, 2059.

(15) Meisel, D.; Czapski, G.; Matheson, M. S.; Mulac, W. A. Int. J. Radiat. Phys. Chem. 1975, 7, 233. Telser, Th.; Shindewolf, U. J. Phys. Chem. 1986, 90, 5378 .

(16) Schmidt, K. H.; Bartels, D. M. Chem. Phys. 1995, 190, 145.

(17) Kirkwood, J. G. In Theory of Liquids; Alder, B. J., Ed.; Gordon and Breach: New York, 1968.

(18) Toukan, K.; Rahman, A. Phys. Rev. B 1985, 31, 2643.

(19) Rossky, P. J.; Schnitker, J. J. Phys. Chem. 1988, 92, 4277.

(20) Turi, L.; Borgis, D. J. Chem. Phys. 2002, 117, 6186.

(21) The Schnitker-Rossky pseudopotential (ref 19) contains a term representing the polarizability of each water molecule, but neglects dispersion. Dispersion has been found to contribute significantly to the energetics of small anionic water clusters [Jordan, K. D.; Wang, F. Annu. Rev. Phys. Chem. 2003, 54, 367]; however, we do not expect dispersion to be important for hydrated electrons or dielectrons in bulk water because the dispersion terms have a magnitude that is inversely proportional to the energy difference between the occupied and unoccupied electronic states. This energy difference is $\sim 10 \mathrm{meV}$ for small anionic water clusters, leading to a net dispersion energy of order $\sim 10 \mathrm{meV}$. In contrast, hydrated electrons and dielectrons have energy differences between the ground and excited states of order $\sim 1 \mathrm{eV}$, which suggests that each water molecule in the first solvation shell should contribute only $\sim 0.1 \mathrm{meV}$ of dispersion to the total energy. Thus, the dispersion energy of hydrated electrons and dielectrons is small enough to be safely neglected in our calculations.

(22) Allen, M. P.; Tildesley, D. J. Computer Simulation of Liquids; Oxford University Press: London, UK, 1992.

(23) Steinhauser, O. Mol. Phys. 1982, 45, 335.

(24) Webster, F.; Rossky, P. J.; Friesner, R. A. Comp. Phys. Comm 1991, 63, 494.

(25) Prezhdo, O. V.; Rossky, P. J. J. Chem. Phys. 1997, 107, 825.

(26) Larsen, R. E.; Schwartz, B. J. J. Phys. Chem., in press.

(27) Because of the cyclical symmetry of the trace operation, the mixed quantum/classical version of thermodynamic integration turns out to be identical with ordinary classical thermodynamic integration.

(28) By averaging in this fashion, we have performed multicanonical TI, which is not as subject to bias as methods which continuously evolve $\lambda$ throughout a single simulation run.

(29) For triplet hydrated dielectrons, the total energy $E_{\mathrm{QM}}+V_{\mathrm{aq}}=$ -87.33(0.64), with $E_{\mathrm{QM}}=-4.71(0.22)$, as reported in Table 1 of ref 13 .

(30) CRC Handbook of Chemistry and Physics, 71st ed.; Lide, D. R., Ed.; CRC Press: Boca Raton, FL, 1990; p 6-96.

(31) Han, P.; Bartels, D. M. J. Phys. Chem. 1990, 94, 7294.

(32) The reference state used for the experimental determination of the hydration free energy of the electron (ref 31) is the hydrated proton. We are not aware of any calculation of the thermodynamic properties of this state for SPC/Flex water relative to our reference state of neat water, however, and we do not believe that a classical treatment of the proton will suffice due to neglect of proton tunneling. Therefore, we cannot compare our free energy differences directly to those reported in ref 31 .

(33) The twenty injection runs were performed using ten statistically independent initial configurations from a ground-state, adiabatic molecular dynamics trajectory for a single hydrated electron. For each configuration, the nonadiabatic relaxation was calculated as described in refs 12 and 26 , using two different random number seeds.

(34) Valleau, J. P.; Card, D. N. J. Chem. Phys. 1972, 57, 5457.

(35) Borgis, D.; Staib, A. J. Chem. Phys. 1996, 104, 4776.

(36) To separate the two electrons in a dielectron by applying a bias potential, one must be able to correct for the bias in forming averages of quantities obtained with the bias, as is done in umbrella sampling (ref 34). For umbrella sampling to apply for a bias potential that acts on quantum degrees of freedom, the bias potential must commute with the Hamiltonian of the system. Most bias potentials do not commute with the kinetic energy operator, so a new approach to quantum umbrella sampling is needed. The form of quantum umbrella sampling derived in ref 35 applies the umbrella potential to the classical degrees of freedom, so the issue of commutativity does not apply, but to separate the two electrons in a dielectron, there is no obvious classical coordinate that can be biased so another approach must be taken (ref 37).

(37) Larsen, R. E.; Schwartz, B. J. Manuscript in preparation.

(38) In the actual calculations, we took advantage of the pairwise-additive nature of the classical and quantum-mechanical forces in our simulations and used a form of eq 8 that is independent of the origin of coordinates, as described in ref 22

(39) Callen, H. B. Thermodynamics and an Introduction to Thermostatistics; John Wiley and Sons: New York, 1985.

(40) Landau, L. D.; Lifshitz, E. M. Statistical Physics, 3rd ed.; Butterworth-Heinemann: Oxford, UK, 1980; Part 1, Section 116.

(41) Huang, C. C.; Couch, G. S.; Pettersen, E. F.; Ferrin, T. E. Pac. Symp. Biocomput. 1996, 1:724. The Chimera code is freely available on the worldwide web at http://www.cgl.ucsf.edu/chimera. 